\title{
Dual-time-point F-18 FDG PET/CT imaging for differentiating the lymph nodes between malignant lymphoma and benign lesions
}

\author{
Michihiro Nakayama $\cdot$ Atsutaka Okizaki $\cdot$ \\ Shunta Ishitoya $\cdot$ Miki Sakaguchi $\cdot$ Junichi Sato $\cdot$ \\ Tamio Aburano
}

Received: 16 July 2012/ Accepted: 14 November 2012/Published online: 28 November 2012

(C) The Author(s) 2012. This article is published with open access at Springerlink.com

\begin{abstract}
Purpose The purpose of the present study is to evaluate the clinical value of dual-time-point F-18 FDG PET/CT imaging to differentiate malignant lymphoma (ML) from benign lymph node (BLN).

Materials and methods The subjects were 310 lymph nodes in 84 patients (195 ML lesions in 30 patients and 115 BLN in 54 patients associated with various etiologies.). F-18 FDG PET/CT scan was performed at 50 min (early scan) and at $100 \mathrm{~min}$ (delayed scan) after the injection. First, the maximum standardized uptake value (SUVmax) of each lesion at early and delayed scans was calculated. Second, we estimated the difference between early and delayed SUVmax (D-SUVmax) and the retention index (RI-SUVmax) to evaluate the change of tracers in the lesions. Furthermore, proper cut-off values of them were evaluated using receiver operating characteristic analysis. The efficacy of each parameter was analyzed with ANOVA.

Results Delayed SUVmax and D-SUVmax in ML were significantly higher than those in BLN. Proper cut-off value in delayed SUVmax was 4.0 and in D-SUVmax was 1.0. When the proper cut-off value in D-SUVmax was applied, the D-SUVmax yielded the role of diagnosis with sensitivity of $82.6 \%$, specificity of $65.2 \%$, positive predictive value of $80.1 \%$ and negative predictive value of $68.8 \%$, respectively.
\end{abstract}

M. Nakayama $(\varangle) \cdot$ A. Okizaki · S. Ishitoya $\cdot$ T. Aburano Department of Radiology, Asahikawa Medical University, 2-1-1-1 Midorigaoka-higashi, Asahikawa 078-8510, Japan e-mail: m-naka@asahikawa-med.ac.jp

M. Sakaguchi · J. Sato

Division of Radiology, Asahikawa Medical University Hospital, Asahikawa, Japan
Conclusions The delayed SUVmax and D-SUVmax were useful indices to differentiate ML from BLN, regardless of histologic subtype. Dual-time-point F-18 FDG PET/CT imaging may help to consider whether there is any need to proceed to more invasive tests, such as biopsy, in individual patients.

Keywords 18F-FDG PET/CT .

Dual-time-point imaging · Lymphoma ·

Benign lymph node $\cdot$ Standardized uptake value

\section{Introduction}

Malignant lymphoma (ML) is one of the most common hematologic malignancy [1]. According to the National Cancer Institute in the US, there were 75,190 new cases of the malignant lymphomas in the US in 2011, and 20,620 deaths from the diseases. Fluorine-18-fluorodeoxyglucose (F-18 FDG) positron emission tomography/computed tomography (PET/CT) is widely used for staging and treatment evaluation of ML [2, 3]. PET/CT offers advantages over conventional imaging techniques, because it can demonstrate abnormal metabolic activity and extension of malignant diseases [4-6]. However, many inflammatory lesions such as pneumonia, sarcoidosis, rheumatoid arthritis, etc., also have elevated F-18 FDG uptake in PET, leading to false-positive results [7-10]. Moreover, there is considerable overlap between the maximum standardized uptake values (SUVs) of ML and BLN, causing difficulty in correctly diagnosing F-18 FDG PET data [11-13]. Some reports indicated that F-18 FDG PET was sensitive and specific in the diagnosis and staging for patients with ML on single-time-point scans $[14,15]$. In patients with head and neck malignant tumors [16], pulmonary nodules [17, 18], 
breast cancer [19], pancreas tumors [20], and bone malignant tumor [21], several authors have reported significant improvement in the diagnostic accuracy of FDG PET scan with dual-time-point scans.

F-18 FDG uptakes in many malignant lesions are increased with time interval [22, 23]. In patients with ML, recently, Shinya et al. [2] statistically evaluated the differences of FDG uptake in early and delayed scans, and compared the maximum SUV (SUVmax) and the retention index (RI) of SUVmax (RI-SUVmax) between the different grades of lymphoma. Because various cell types exhibit varying rates of F-18 FDG uptake [16, 17, 19, 23, 24], we hypothesized that measurements of SUVmax at two time points may prove to be of value in differentiating ML from BLN.

Therefore, the purpose of this study was to assess the usefulness of dual-time-point F-18 FDG PET/CT scan in initial diagnosis of ML, with analyses of the SUVmax on 50-min early and 100-min delayed scans.

\section{Materials and methods}

\section{Patient population}

The subjects consisted of 30 patients ( 15 men and 15 women; mean age, $64.6 \pm 12.2$ years) with 195 lymph nodes of ML and 54 patients (26 men and 28 women; mean age, $61.7 \pm 17.7$ years) with 115 benign lymph nodes associated with various etiologies. Patient characteristics are summarized in Table 1. All patients underwent F-18 FDG PET/CT scanning for the differential diagnosis from July 2009 to May 2011. They were finally diagnosed according to clinical and radiologic follow-up (follow-up period of time; at least 6 months) and/or histopathological findings. The histopathology examinations confirmed 30 patients with ML and 29 patients with BLN. 25 patients with BLN were finally diagnosed according to clinical and radiologic follow-up $(8.74 \pm 3.30$ months, range 6-18 months). Reactive lymph nodes are defined as lymph nodes, which diagnosed no malignancy through a clinical follow-up with no treatment or proof of no increase in size and accumulations on F-18 FDG PET/CT scans, in the patients with suspected malignancy. None of the patients had received chemotherapy or radiation therapy before the dual-time-point F-18 FDG PET scans. Informed consent was obtained from all patients participating in the study. This study was retrospective and ethics committee approval was deemed unnecessary in our institution.

\section{F-18 FDG PET/CT scans}

All imaging and data acquisition was performed on a combined PET/CT in-line system (Discovery VCT, GE
Healthcare, Milwaukee, WI, USA). This device integrates a PET scanner with a 64 multi-detector row $\mathrm{CT}$ and permits the acquisition of coregistered CT and PET images in the same session. At the time of F-18 FDG injection, all patients had fasted for at least $4 \mathrm{~h}$ and had blood sugar levels of less than $120 \mathrm{mg} / \mathrm{dl}$. The scan was performed twice: an early whole-body (from head to pelvis) scan at $50 \mathrm{~min}$ after the injection of $3.7 \mathrm{MBq} / \mathrm{kg}$ of F-18 FDG, followed by a delayed scan at $100 \mathrm{~min}$. The scans consisted of 7 or 8 beds, and the length of one bed was $16 \mathrm{~cm}$. A delayed scan was performed for the same field of view and scan area in early scan. The image acquisition time per bed was $2-3 \mathrm{~min}$. The PET images were reconstructed iteratively with three dimensional ordered-subset expectation maximization (VUE point, GE Healthcare, Milwaukee, WI, USA). An unenhanced CT scan (100 mA with auto $\mathrm{mA}, 0.6 \mathrm{~s} /$ rotation, $120 \mathrm{kV}$ ) from the head to the pelvic floor was acquired (reconstructed slice thickness $3.27 \mathrm{~mm}$ ) for fusing with PET images. PET/CT fusion images on three orthogonal (transaxial, coronal and sagittal) planes were reviewed on a workstation (Advantage Workstation version 4.4, GE Healthcare, Milwaukee, WI, USA).

Image analysis

F-18 FDG accumulations were considered positive when focal uptake was more intense than the mediastinal bloodpool activity in the early scan or delayed scan. PET images were interpreted independently and prospectively by two experienced nuclear medicine physicians, without the knowledge of histopathologic or other radiologic data. In case of a discrepant reading, the two physicians discussed the case together to reach consensus about referral. The results of FDG uptake were classified by a four-score visual scale using the individuals' blood-pool radioactivity as a reference. The classification was defined as follows: 0 radioactivity lower than that of blood-pool; 1 radioactivity equal to that of bloodpool; 2 radioactivity mild higher than that of blood-pool; and 3 radioactivity obviously higher than that of blood-pool. The short-axis diameter of lymph nodes was measured on CT scan. The maximum standardized uptake value (SUVmax) of lymph node-related F-18 FDG accumulation on $50 \mathrm{~min}$ (early SUVmax) and 100 min (delayed SUVmax) images after F-18 FDG injection was calculated as follows:

$\begin{aligned} \text { SUV }= & \{\text { Tissue activity }(\mathrm{Bq} / \mathrm{g})\} /\{\text { Injected } \mathrm{F} \\ & -18 \text { FDG dose }(\mathrm{Bq}) / \text { body weight }(\mathrm{g})\}\end{aligned}$

A region of interest (ROI) which was manually drawn for SUVmax calculation on early PET scans was placed over the area of maximal metabolic activity on the transaxial slice showing tumor-related increased uptake. On delayed PET scans, ROIs were placed in identical positions. In addition to RI-SUVmax, we tried to evaluate 
Table 1 Characteristics of malignant lymphoma and benign lymph nodes

\begin{tabular}{|c|c|c|c|c|c|c|}
\hline & $\begin{array}{l}\text { Number of } \\
\text { patients }\end{array}$ & $\begin{array}{l}\text { Number of } \\
\text { lesions }\end{array}$ & $\begin{array}{l}\text { Early SUVmax } \\
(\text { mean } \pm \text { SD) }\end{array}$ & $\begin{array}{l}\text { Delayed SUVmax } \\
(\text { mean } \pm \text { SD })\end{array}$ & $\begin{array}{l}\text { D-SUVmax } \\
(\text { mean } \pm \text { SD) }\end{array}$ & $\begin{array}{l}\text { RI-SUVmax } \\
(\text { mean } \pm \text { SD) }\end{array}$ \\
\hline \multicolumn{7}{|l|}{ Malignant lymphoma ${ }^{\mathrm{a}}$} \\
\hline \multicolumn{7}{|c|}{ Histological subtypes of lymphoma } \\
\hline $\begin{array}{l}\text { Diffuse large B-cell } \\
\text { lymphoma }\end{array}$ & 12 & 74 & $7.24 \pm 6.18$ & $9.35 \pm 7.05$ & $2.11 \pm 1.41$ & $0.42 \pm 0.24$ \\
\hline Follicular lymphoma & 10 & 70 & $8.13 \pm 5.75$ & $10.18 \pm 6.80$ & $2.05 \pm 1.56$ & $0.32 \pm 0.23$ \\
\hline T-cell lymphoma & 5 & 34 & $4.19 \pm 2.58$ & $5.79 \pm 3.01$ & $1.60 \pm 0.87$ & $0.43 \pm 0.19$ \\
\hline Hodgkin's lymphoma & 2 & 13 & $6.44 \pm 3.57$ & $8.06 \pm 3.67$ & $1.62 \pm 0.67$ & $0.30 \pm 0.13$ \\
\hline Mantle cell lymphoma & 1 & 4 & $4.18 \pm 0.62$ & $5.28 \pm 0.66$ & $1.10 \pm 0.19$ & $0.27 \pm 0.07$ \\
\hline Total & 30 & 195 & $6.70 \pm 5.43$ & $8.62 \pm 6.27$ & $1.91 \pm 1.33$ & $0.38 \pm 0.23$ \\
\hline \multicolumn{7}{|l|}{ Benign lymph nodes ${ }^{\mathrm{b}}$} \\
\hline \multicolumn{7}{|l|}{ Disorders } \\
\hline Reactive lymph node & 24 & 54 & $3.03 \pm 0.90$ & $3.69 \pm 1.02$ & $0.65 \pm 0.52$ & $0.23 \pm 0.20$ \\
\hline Lymphadenitis & 10 & 20 & $3.88 \pm 3.06$ & $5.34 \pm 4.04$ & $1.47 \pm 2.00$ & $0.34 \pm 0.40$ \\
\hline Pneumonia & 9 & 16 & $2.88 \pm 0.74$ & $3.71 \pm 1.09$ & $0.82 \pm 0.63$ & $0.29 \pm 0.20$ \\
\hline Rheumatoid arthritis & 3 & 9 & $3.26 \pm 1.32$ & $3.62 \pm 1.15$ & $0.37 \pm 0.31$ & $0.16 \pm 0.16$ \\
\hline Sarcoidosis & 2 & 5 & $7.60 \pm 4.37$ & $8.26 \pm 4.80$ & $0.66 \pm 0.89$ & $0.09 \pm 0.10$ \\
\hline Silicosis & 1 & 5 & $2.60 \pm 0.39$ & $3.66 \pm 0.72$ & $1.06 \pm 0.60$ & $0.41 \pm 0.27$ \\
\hline IgG4-related disease & 1 & 2 & $3.50 \pm 1.00$ & $3.80 \pm 1.30$ & $0.30 \pm 0.30$ & $0.07 \pm 0.07$ \\
\hline Tuberculosis & 1 & 1 & 2.60 & 3.20 & 0.60 & 0.23 \\
\hline Echinococcus & 1 & 1 & 4.50 & 5.60 & 1.10 & 0.24 \\
\hline Benign lung tumor & 1 & 1 & 1.50 & 1.90 & 0.40 & 0.27 \\
\hline Benign brain tumor & 1 & 1 & 4.40 & 4.80 & 0.4 & 0.09 \\
\hline Total & 54 & 115 & $3.37 \pm 2.43$ & $4.16 \pm 2.44$ & $0.80 \pm 1.04$ & $0.25 \pm 0.25$ \\
\hline
\end{tabular}

a Size of lesions: $1.2 \mathrm{~cm} \pm 0.8$ in short-axis diameter, range $0.3-2.7 \mathrm{~cm}$

b Size of lesions: $1.0 \mathrm{~cm} \pm 0.5$ in short-axis diameter, range $0.3-2.4 \mathrm{~cm}$

an usefulness of difference between early SUVmax and delayed SUVmax (D-SUVmax) in this study, as a more simple index. The RI-SUVmax and the D-SUVmax were calculated as follows:

$$
\begin{aligned}
\text { RI-SUVmax }= & (\text { Delayed SUVmax }- \text { Early SUVmax }) / \\
& \text { Early SUVmax } \\
\text { D-SUVmax = } & \text { Delayed SUVmax }- \text { Early SUVmax }
\end{aligned}
$$

We estimated these quantitative values to evaluate the change of tracers in the lesions at 50 and $100 \mathrm{~min}$ after the F-18 FDG injection.

All lymph nodes showed increased accumulations of F-18 FDG on early and delayed scans. D-SUVmax and RISUVmax were calculated with uptake of same lymph nodes on the both scans.

\section{Statistical analysis}

Differences in visual scores analyzed with the Wilcoxon signed-rank test, using the STATISTICA 03J (StatSoft Inc.,
Tulsa, OK, USA). All data were expressed as mean \pm standard deviation (SD). Receiver operating characteristic (ROC) analysis was performed to determine the proper cutoff value for the difference ML and BLN. The overall statistically difference of area under the curves (AUCs) was evaluated with ANOVA, and we compared differences between AUCs of those indices using DBM-MRMC, version 2.2, software (http://perception.radiology.uiowa.edu). A paired 2-tailed $t$ test was used to compare differences between ML and BLN in those indices. $P$ values of less than 0.05 were considered statistically significant.

\section{Results}

ROC analysis

The detailed data are described in Table 2. ROC analysis revealed that the use of delayed SUVmax and D-SUVmax provided better differentiation between ML and BLN than the use of early SUVmax and RI-SUVmax alone (Fig. 1). 
Table 2 Diagnostic values for differentiation of ML and BLN with parameters of dual-time-point PET/CT scan

\begin{tabular}{lllll}
\hline & $\begin{array}{l}\text { Cut-off } \\
\text { value }\end{array}$ & $\begin{array}{l}\text { Sensitivity/ } \\
\text { specificity }(\%)\end{array}$ & $\begin{array}{l}\text { PPV/NPV } \\
(\%)\end{array}$ & AUC \\
\hline $\begin{array}{l}\text { Early } \\
\quad \text { SUVmax }\end{array}$ & 3.0 & $70.8 / 58.2$ & $74.2 / 56.8$ & 0.715 \\
$\begin{array}{c}\text { Delayed } \\
\quad \text { SUVmax }\end{array}$ & 4.0 & $75.4 / 60.0$ & $76.2 / 61.0$ & $0.772^{*}$ \\
$\begin{array}{l}\text { D-SUVmax } \\
\text { RI-SUVmax }\end{array}$ & 1.0 & $82.6 / 65.2$ & $80.1 / 68.8$ & $0.809^{*}$ \\
\hline
\end{tabular}

$P P V$ positive predictive value, $N P V$ negative predictive value, $A U C$ area under the curve

$* P<0.005$

The AUCs in delayed SUVmax and D-SUVmax were statistically greater than in early SUVmax $(P<0.005)$. There was no significant difference between the AUCs in delayed SUVmax and D-SUVmax. The largest AUC has obtained with D-SUVmax in the four indices. The proper cut-off value of D-SUVmax for the differential diagnosis was $>1.0 ; 161$ of $195 \mathrm{ML}$ and 75 of 115 BLN were correctly diagnosed using the cut-off value, yielding a sensitivity of $82.6 \%$, specificity of $65.2 \%$, positive predictive value (PPV) of $80.1 \%$, and negative predictive value (NPV) of $68.8 \%$ (Table 2). The accuracy of D-SUVmax was $76.1 \%$, and the highest value compared with other indices or its combination.

\section{SUVmax and RI-SUVmax}

The mean values of early SUV max, delayed SUVmax, D-SUVmax, and RI-SUVmax were $6.70 \pm 5.43,8.62 \pm$ $6.27,1.91 \pm 1.33$ and $0.38 \pm 0.23$ in ML, and $3.37 \pm$ $2.43,4.16 \pm 2.44,0.80 \pm 1.04$ and $0.25 \pm 0.25$ in BLN, respectively. There were significant differences between ML and BLN in those indices $(P<0.01$ for all comparisons; Figs. 2, 3). ML and BLN tended to demonstrate increase uptake in visual scores on delayed scans, but there was no significant difference between ML and BLN (Table 3).

\section{Discussion}

The present study evaluated the usefulness of SUVmax and RI-SUVmax on dual-time-point F-18 FDG PET/CT scans, performed at 50 and $100 \mathrm{~min}$ after tracer injection, in patients with ML and BLN. The AUCs of delayed SUVmax and D-SUVmax were statistically greater than that of early SUVmax. D-SUVmax had the largest AUC among the four indices, which led to improved sensitivity and specificity.

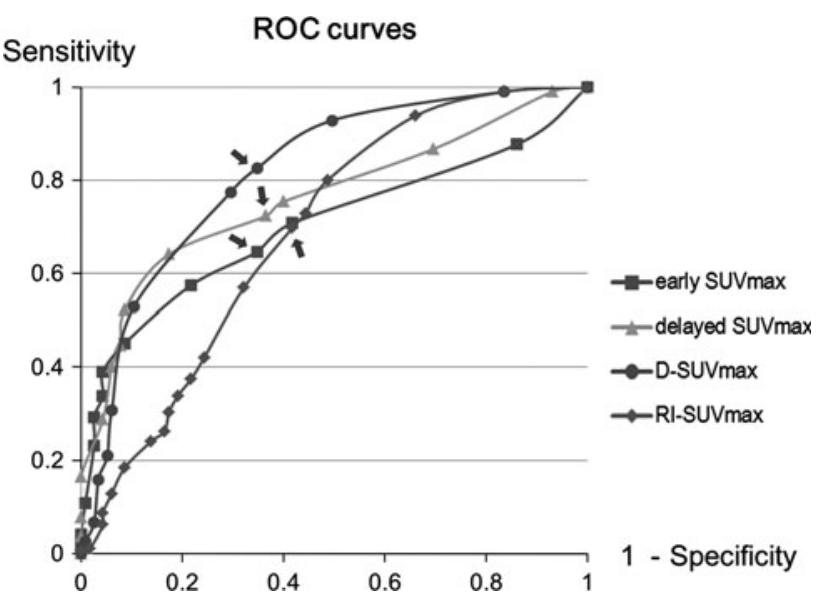

Fig. 1 ROC analysis showed that the D-SUVmax provided a best statistical differentiation between ML and BLN. ROC analysis revealed that the proper cut-off values of early, delayed, D- and RI-SUVmax were 3.0, 4.0, 1.0 and 0.22, respectively (arrows)

The F-18 FDG uptake by lymphoma is probably associated with Glut1 expression [25, 26] and Ki-67 values $[27,28]$. In many malignant tumors, scan start times of 45-60 min have been reported to cause significant underestimation of the true SUVs, because SUVs do not reach maximum levels until several hours after F-18 FDG injection [2, 23, 29]. Kumar et al. reported that F-18 FDG uptake in malignant cells was related to low glucose-6phosphatase activity, and increased glucose uptake through glucose transporter proteins in these cells. In contrast, such a prolonged period of F-18 FDG uptake is rare in benign lesions or normal tissues [22, 23]. Gupta et al. [30] reported that the influx rate constant was greater in malignant lesions than in benign lesions, and that continuous tracer uptake by malignant lesions was observed in pulmonary tumor.

According to our results, delayed SUVmax and D-SUVmax were significantly better predictors of ML than early SUVmax. The AUC of D-SUVmax was the greatest among these four indices. Dual-time-point scans may have an advantage to observe the serial change of uptake in the lesions. Contrary to expectations, the present study showed that there was no significant difference in AUC between RI-SUVmax and early SUVmax. In principle, RI-SUVmax must have a merit of not being dependent on scale and is a useful index [2, 31]. Some authors reported RI is a good predictor for diagnosis and prognosis, and is superior to only early imaging $[32,33]$. The reason is unknown why RI-SUVmax was not the best predictor, but our results are consistent with that of a previous study [18].

Some institutions, which give a high priority to economic requirements and protection of excessive radiation exposure, may perform only a single scan. In the $t$ test to compare differences between ML and BLN, there was a 


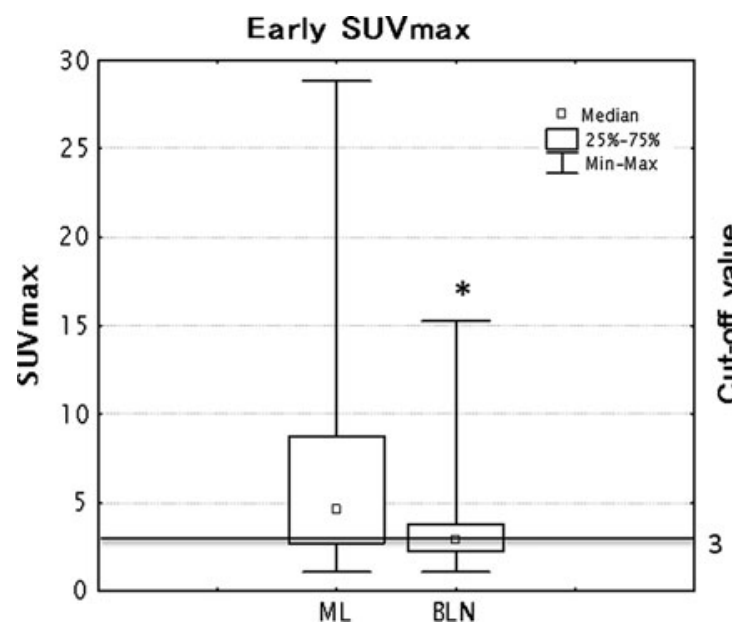

Fig. 2 Box and whiskers plots showing distribution of early and delayed SUVmax among ML and BLN. The graph showed the distribution of data based on the five number summary: minimum,

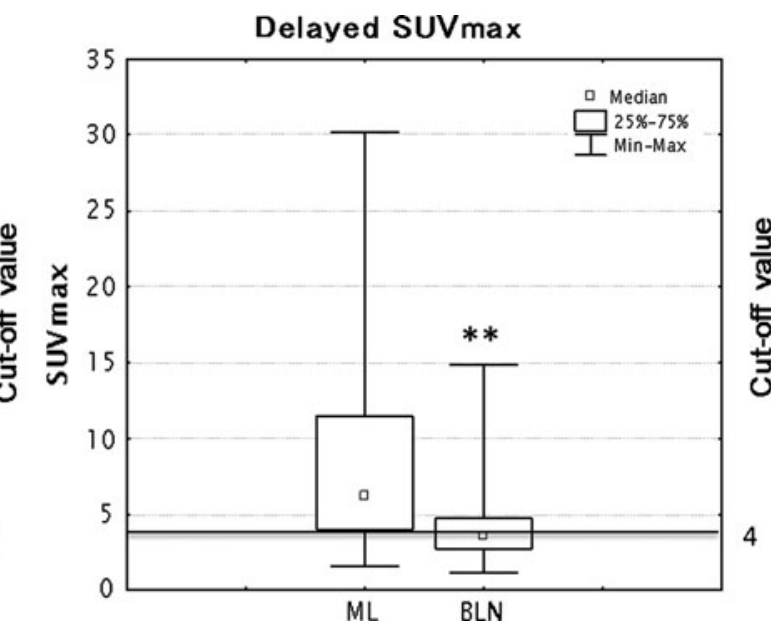

first quartile, median, third quartile, and maximum $(* P<0.01$, compared with early SUVmax in ML, $* * P<0.01$, compared with delayed SUVmax in ML)
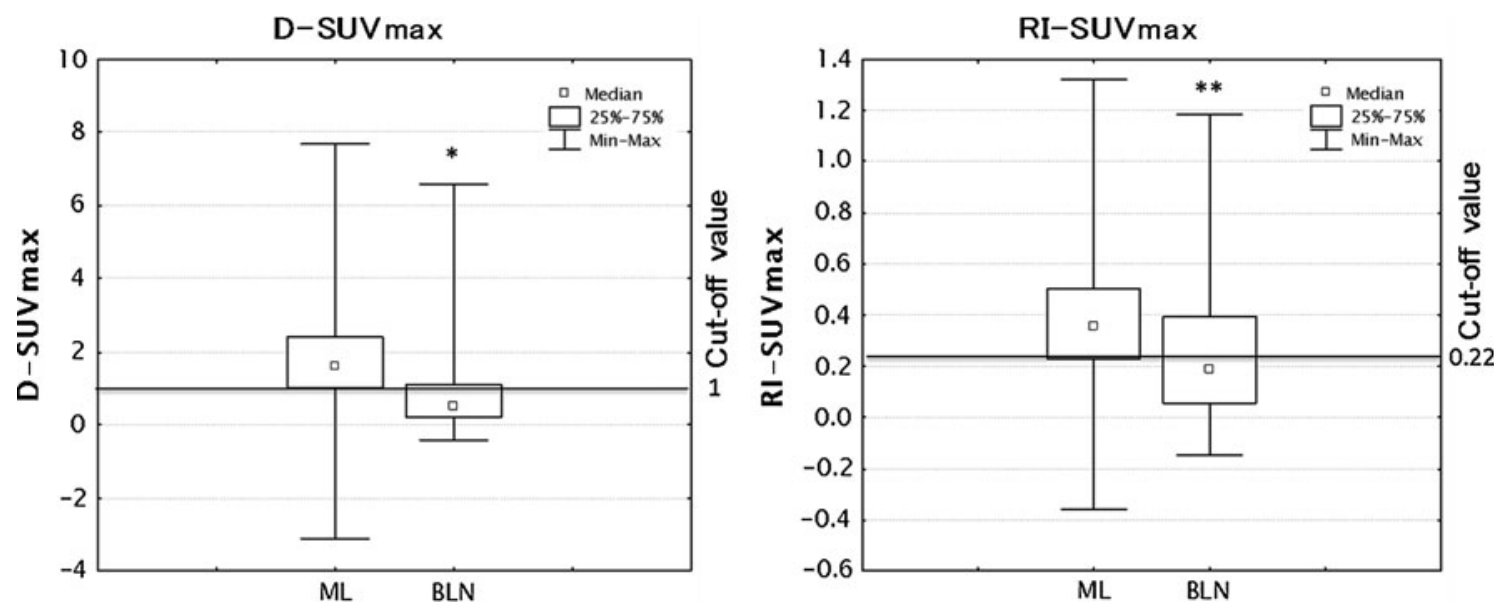

Fig. 3 Box and whiskers plots showing distribution of D-SUVmax and RI-SUVmax among ML and BLN. The graph showed the distribution of data as mentioned in Fig. $2(* P<0.01$, compared with D-SUVmax in ML, $* * P<0.01$, compared with RI-SUVmax in ML)

Table 3 Visual scoring results: ML versus BLN on dual-time-point scan

\begin{tabular}{lcccc}
\hline $\begin{array}{l}\text { Visual } \\
\text { score }\end{array}$ & $\begin{array}{l}\text { ML (early } \\
\text { scan) }\end{array}$ & $\begin{array}{l}\text { BLN (early } \\
\text { scan) }\end{array}$ & $\begin{array}{l}\text { ML (delayed } \\
\text { scan) }\end{array}$ & $\begin{array}{l}\text { BLN (delayed } \\
\text { scan) }\end{array}$ \\
\hline 0 & 20 & 16 & 18 & 9 \\
1 & 38 & 32 & 33 & 42 \\
2 & 118 & 57 & 120 & 54 \\
3 & 19 & 10 & 24 & 19 \\
Total & 195 & 115 & 195 & 115 \\
\hline
\end{tabular}

significant difference between ML and BLN in all indices. According to this result, it may be preferable to use the early SUVmax cut-off value to avoid increased radiation exposure. However, obtaining these dual-time-point scans might have advantages such as discrimination of mesenteric lymph nodes from physiological intestinal accumulations [34] and discrimination of malignant tumors from benign tumors [17-22]. Focal FDG foci due to urinary excretion in the ureter can be mistaken as lymphadenopathy close to the ureter, such as ovary, cervix, pancreatic tail and adrenal grand [9, 35]. Co-registration of PET with anatomical imaging may be useful to avoid misinterpretation; however, it is still difficult to differentiate lesions in proximity to urinary activity, especially in a PET/CT study acquired without an intravenous contrast agent. In contrast, additional delayed scanning is a simple option [36]. In addition, the use of delayed SUVmax and D-SUVmax might improve the sensitivity and specificity for diagnosis of ML.

This study had several limitations. First, the subject population comprised a relatively small number of patients. The smallest group had only one patient, and the 
small numbers of subjects disturbed the analysis of differences in each histological subtype. Consequently, we performed ROC analysis without comparing each histological subtype. However, some authors have reported that the SUVmax tended to be lower in Hodgkin's lymphoma and T-cell lymph nodes than in B-cell lymphoma, with differences observed in the early SUVmax [28]. Examination of a larger number of patients in a prospective study could help in evaluating the role of dualtime-point PET/CT scans in diagnosis of lymphoma. Second, the cut-off value obtained in this study was based on the data collected at our institution alone, and the absolute value of SUVmax may vary according to the imaging system used. We intend to address this issue by conducting similar comparisons at multiple institutions in future. Finally, histopathological examination of biopsy samples from all lymph nodes suspected of having F-18 FDG uptake on PET/CT was not performed. However, despite these limitations, our results suggest that the use of dual-time-point PET/CT scans might improve diagnostic capability for lymphoma compared with conventional techniques.

\section{Conclusion}

In conclusion, dual-time-point F-18 FDG PET/CT scan is useful for differential diagnosis between ML and BLN, regardless of a variety of the histologic subtype. Dual-timepoint F-18 FDG PET/CT imaging may help to consider whether there is any need to proceed to more invasive tests, such as biopsy, in individual patients.

Conflict of interest The authors declare that they have no conflict of interest.

Open Access This article is distributed under the terms of the Creative Commons Attribution License which permits any use, distribution, and reproduction in any medium, provided the original author(s) and the source are credited.

\section{References}

1. Jhanwar YS, Straus DJ. The role of PET in lymphoma. J Nucl Med. 2006;47:1326-34.

2. Shinya T, Fujii S, Asakura S, Taniguchi T, Yoshio K, Alafate A, et al. Dual-time-point F-18 FDG PET/CT for evaluation in patients with malignant lymphoma. Ann Nucl Med. 2012;13 [Epub ahead of print].

3. Ahmadzadehfar H, Rodrigues M, Zakavi R, Knoll P, Mirzaei S. Prognostic significance of the standardized uptake value of pretherapeutic (18)F-FDG PET in patients with malignant lymphoma. Med Oncol. 2011;28:1570-6.

4. Kakhki VR. Positron emission tomography in the management of lung cancer. Ann Thorac Med. 2007;2:69-76.
5. Schrepfer T, Haerle SK, Strobel K, Schaefer N, Hälg RA, Huber GF. The value of (18)F-fluorodeoxyglucose positron emission tomography/computed tomography for staging of primary extranodal head and neck lymphomas. Laryngoscope. 2010;120: 937-44.

6. Kapoor V, McCook BM, Torok FS. An introduction to PET-CT imaging. Radiographics. 2004;24:523-43.

7. Tamura M, Oda M, Matsumoto I, Waseda R, Watanabe G. Pattern and predictors of false positive lymph node involvement on positron emission tomography in patients with non-small cell lung cancer. Thorac Cardiovasc Surg. 2012;60:105-10.

8. Castellucci P, Zinzani P, Pourdehnad M, Alinari L, Nanni C, Farsad M, et al. 18F-FDG PET in malignant lymphoma: significance of positive findings. Eur $\mathrm{J}$ Nucl Med Mol Imaging. 2005;32:749-56

9. Shreve PD, Anzai Y, Wahl RL. Pitfalls in oncologic diagnosis with FDG PET imaging: physiologic and benign variants. Radiographics. 1999;19:61-77.

10. Zhuang H, Pourdehnad M, Lambright ES, Yamamoto AJ, Lanuti M, Li P, et al. Dual-time-point 18F-FDG PET imaging for differentiating malignant from inflammatory processes. J Nucl Med. 2001;42:1412-7.

11. Sonet A, Graux C, Nollevaux MC, Krug B, Bosly A, Vander Borght T. Unsuspected FDG-PET findings in the follow-up of patients with lymphoma. Ann Hematol. 2007;86:9-15.

12. Zinzani PL, Tani M, Trisolini R, et al. Histological verification of positive positron emission tomography findings in the follow-up of patients with mediastinal lymphoma. Haematologica. 2007;92: $771-7$.

13. Maayan H, Ashkenazi Y, Nagler A, Izbicki G. Sarcoidosis and lymphoma: case series and literature review. Sarcoidosis Vasc Diffuse Lung Dis. 2011;28:146-52.

14. Isasi CR, Lu P, Blaufox MD. A metaanalysis of 18F-2-deoxy-2fluori-D-glucose positron emission tomography in the staging and restaging of patients with lymphoma. Cancer. 2005;104:1066-74.

15. Zijlstra JM, Lindauer-van der Werf G, Hoekstra OS, Hooft L, Riphagen II, Huijgens PC. 18F-fluoro-deoxyglucose positron emission tomography for post-treatment evaluation of malignant lymphoma: a systematic review. Haematologica. 2006;91:522-9.

16. Hustinx R, Smith RJ, Benard F, Rosenthal DI, Machtay M, Farber LA, et al. Dual-time-point fluorine-18 fluorodeoxyglucose positron emission tomography: a potential method to differentiate malignancy from inflammation and normal tissue in the head and neck. Eur J Nucl Med. 1999;26:1345-8.

17. Matthies A, Hickeson M, Cuchiara A, Alavi A. Dual time point 18F-FDG PET for the evaluation of pulmonary nodules. J Nucl Med. 2002;43:871-5.

18. Suga K, Kawakami Y, Hiyama A, Sugi K, Okabe K, Matsumoto $\mathrm{T}$, et al. Differential diagnosis between (18)F-FDG-avid metastatic lymph nodes in non-small cell lung cancer and benign nodes on dual-time-point PET/CT scan. Ann Nucl Med. 2009; 23:523-31.

19. Mavi A, Urhan M, Yu JQ, Zhuang H, Houseni M, Cermik TF, et al. Dual-time-point 18F-FDG PET imaging detects breast cancer with high sensitivity and correlates well with histologic subtypes. J Nucl Med. 2006;47:1440-6.

20. Nakamoto Y, Higashi T, Sakahara H, Tamaki N, Kogire M, Doi $\mathrm{R}$, et al. Delayed (18)F-fluoro-2-deoxy-D-glucose positron emission tomography scan for differentiation between malignant and benign lesions in the pancreas. Cancer. 2000;89:2547-54.

21. Tian R, Su M, Tian Y, Li F, Li L, Kuang A, et al. Dual-time point PET/CT with F-18 FDG for the differentiation of malignant and benign bone lesions. Skeletal Radiol. 2009;38:451-8.

22. Kumar R, Loving VA, Chauhan A, Zhuang H, Mitchell S, Alavi A. Potential of dual-time-point imaging to improve breast cancer diagnosis with (18)F-FDG PET. J Nucl Med. 2005;46:1819-24. 
23. Hamberg LM, Hunter GJ, Alpert NM, Choi NC, Babich JW, Fischman AJ. The dose uptake ratio as an index of glucose metabolism: useful parameter or oversimplification? J Nucl Med. 1994;35:1308-12.

24. Lodge MA, Lucas JD, Marsden PK, Cronin BF, O’Doherty MJ, Smith MA. A PET study of 18FDG uptake in soft tissue masses. Eur J Nucl Med. 1999;26:22-30.

25. Khandani AH, Dunphy CH, Meteesatien P, Dufault DL, Ivanovic $\mathrm{M}$, Shea TC. Glut1 and Glut3 expression in lymphoma and their association with tumor intensity on $18 \mathrm{~F}$-fluorodeoxyglucose positron emission tomography. Nucl Med Commun. 2009;30: 594-601.

26. Bedigian MP, Benard F, Smith RJ, Karp JS, Alavi A. Wholebody positron emission tomography for oncology imaging using singles transmission scanning with segmentation and ordered subsets-expectation maximization (OS-EM) reconstruction. Eur $\mathbf{J}$ Nucl Med. 1998;25:659-61.

27. Papajík T, Mysliveček M, Sedová Z, Buriánková E, Procházka V, Koranda P, et al. Standardised uptake value of $18 \mathrm{~F}-\mathrm{FDG}$ on staging PET/CT in newly diagnosed patients with different subtypes of non-Hodgkin's lymphoma. Eur J Haematol. 2011;86: $32-7$.

28. Wu X, Pertovaara H, Korkola P, Vornanen M, Eskola H, Kellokumpu-Lehtinen PL. Glucose metabolism correlated with cellular proliferation in diffuse large B-cell lymphoma. Leuk Lymphoma. 2011;53:400-5.

29. Kubota K, Itoh M, Ozaki K, Ono S, Tashiro M, Yamaguchi K, et al. Advantage of delayed whole-body FDG-PET imaging for tumour detection. Eur J Nucl Med. 2001;28:696-703.
30. Gupta N, Gill H, Graeber G, Bishop H, Hurst J, Stephens T. Dynamic positron emission tomography with F-18 fluorodeoxyglucose imaging in differentiation of benign from malignant lung/mediastinal lesions. Chest. 1998;114:1105-11.

31. Nagamachi S, Hoshi H, Jinnouchi S, Ohnishi T, Flores LG 2nd, Futami S, Nakahara H, et al. 201TL SPECT for evaluating head and neck cancer. Ann Nucl Med. 1996;10:105-11.

32. Lyshchik A, Higashi T, Nakamoto Y, Fujimoto K, Doi R, Imamura $\mathrm{M}$, et al. Dual-phase 18F-fluoro-2-deoxy-D-glucose positron emission tomography as a prognostic parameter in patients with pancreatic cancer. Eur J Nucl Med Mol Imaging. 2005;32:389-97.

33. Uesaka D, Demura Y, Ishizaki T, Ameshima S, Miyamori I, Sasaki M, et al. Evaluation of dual-time-point 18F-FDG PET for staging in patients with lung cancer. J Nucl Med. 2008;49: 1606-12.

34. Chun H, Kim CK, Krynckyi BR, Machac J. The usefulness of a repeat study for differentiating between bowel activity and local tumor recurrence on FDG PET scans. Clin Nucl Med. 2003;28: $672-3$.

35. Cook GJ, Fogelman I, Maisey MN. Normal physiological and benign pathological variants of 18-fluoro-2-deoxyglucose positron-emission tomography scanning: potential for error in interpretation. Semin Nucl Med. 1996;26:308-14.

36. Miyake KK, Nakamoto Y, Togashi K. Dual-time-point 18F-FDG PET/CT in patients with colorectal cancer: clinical value of early delayed scanning. Ann Nucl Med. 2012;26:492-500. 